\title{
Wavelength modulation spectroscopy: combined frequency and intensity laser modulation
}

\author{
Stéphane Schilt, Luc Thévenaz, and Philippe Robert
}

\begin{abstract}
A theoretical model of wavelength modulation spectroscopy that uses a laser diode on a Lorentzian absorption line is presented. This theory describes the general case of a current-modulated semiconductor laser, for which a combined intensity and frequency modulation with an arbitrary phase shift occurs. On the basis of this model, the effect of several modulation parameters on the detected signals is evaluated. Experimental signals measured on an absorption line of $\mathrm{CO}_{2}$ by use of a $2-\mu \mathrm{m}$ distributedfeedback laser are also presented and validate this analysis. These experimental results agree with the calculated signals, confirming the relevance of the model. (C) 2003 Optical Society of America

OCIS codes: $300.6380,300.6260,300.1030,140.5960$.
\end{abstract}

\section{Introduction}

Wavelength modulation spectroscopy (WMS) is a widely used technique for trace-gas detection by use of semiconductor lasers in the mid-infrared ${ }^{1-7}$ and near infrared. ${ }^{8-13}$ This method takes advantage of several properties of semiconductor lasers, such as their tunability and modulation capabilities, to perform absorbance measurements in the $10^{-5}-10^{-6}$ range. ${ }^{13}$ These performances correspond to detection limits in the parts per $10^{6}$ range for many substances in the near infrared $\left(\mathrm{CO}, \mathrm{CO}_{2}, \mathrm{H}_{2} \mathrm{O}, \mathrm{CH}_{4}\right.$, $\mathrm{NH}_{3}, \mathrm{C}_{2} \mathrm{H}_{2}$, etc.). The detection limit is still improved by 2 or 3 orders of magnitude in the midinfrared. WMS was originally developed by use of lead-salt laser diodes emitting in the mid-infrared. ${ }^{1-4}$ It was then applied to optical communication lasers in the near infrared ${ }^{8-13}$ and more recently to the novel quantum-cascade lasers in the mid-infrared ${ }^{5-7}$ and using visible laser diodes. ${ }^{14}$ WMS is based on the modulation of the light emitted by a laser that is slowly tuned through an absorption feature of the species to be detected. The interaction of the modulated light with the absorption line leads to the generation of signals at different harmonics of the

The authors are with the Laboratory of Metrology and Photonics, Swiss Federal Institute of Technology, École Polytechnique Federal Lausanne, CH-1015, Lausanne, Switzerland. The e-mail address of S. Schilt is stephane.schilt@epfl.ch.

Received 6 February 2003; revised manuscript received 5 July 2003.

0003-6935/03/336728-11\$15.00/0

(C) 2003 Optical Society of America modulation frequency. The signal at a given harmonic can be measured with lock-in detection and is directly proportional to the absorption. An important advantage of this technique is to shift the detection to higher frequencies, at which the laser excess noise $(1 / f$ noise) is reduced.

In a semiconductor laser, a light modulation can be easily performed by modulation of the injection current. This produces a combined frequency modulation (FM) and intensity modulation (IM) of the emitted light, with a phase difference between the two modulations. The IM-FM phase shift depends on the laser structure and modulation frequency, but typical values in distributed-feedback (DFB) lasers decrease from 0 at low frequencies to $-\pi$ at high frequencies. ${ }^{15}$ In WMS the detected signals are mainly due to the FM of the laser emission, whereas IM, often referred as residual amplitude modulation in the literature ${ }^{2}$ is an unwanted effect that distorts the signals. This distortion also depends on the IM-FM phase shift, so that this parameter should be known precisely for a detailed and accurate description of the WMS signals. Whereas the combined IM-FM of laser diodes was well known experimentally for a long time, ${ }^{15,16}$ its contribution to the WMS signals was difficult to take into account theoretically. Theoretical models of WMS have been investigated by several authors, including Arndt, ${ }^{17}$ Reid and Labrie, ${ }^{18}$ and Supplee et al. ${ }^{19}$ Their descriptions did not take into account the associated intensity modulation of the laser but treated only pure FM. The first model taking into account a simultaneous FM and IM was proposed by Philippe and Hanson, ${ }^{20}$ who numerically calculated the $1 f$ and $2 f$ signals by 
using a Fourier decomposition of a Voigt profile. Only recently a generalized and analytical theory of WMS was presented by Kluczynski et al. In a series of papers ${ }^{21-24}$ they developed a complete theory of WMS based on Fourier decomposition of the detected signals. Their model considers the effect of a FM and an associated power modulation. This latter effect includes both the residual amplitude modulation of the laser and background signals produced by multiple reflections in the optical system (etalon effects). Their theory is even capable of handling more complicated situations such as nonlinear IM effects and WMS with frequency-doubled light. ${ }^{23,24}$ Independently of their research and using a slightly different formalism, we have also developed a theoretical model of WMS in the general case of a combined IM-FM with an arbitrary phase shift. The description that we present in this paper uses Arndt's formalism ${ }^{17}$ and generalizes it to take into account the IM of the laser. Analytical expressions for the WMS signals at the first three harmonics are obtained, and the distortion produced by the IM and the IM-FM phase shift in comparison with the case of pure FM is highlighted. In this description the case of a Lorentzian line-shape function is considered. Although this can be considered restrictive at first sight, we are mainly interested in applications of WMS in standard environmental conditions, i.e., at ambient temperature and atmospheric pressure. In such conditions the general Voigt line shape is strongly dominated by the Lorentzian contribution (pressure broadening), whereas the Gaussian (Doppler) contribution can be neglected. The lines are consequently well approximated by a Lorentzian profile, and our model applies to the case of trace-gas detection at atmospheric pressure. The results of this theoretical model are compared with experimental measurements performed on an absorption line of $\mathrm{CO}_{2}$ by use of a DFB laser emitting at $2 \mu \mathrm{m}$. The effect of several parameters on the harmonic signals is considered, and an excellent agreement is obtained between experimental and theoretical signals, validating the developed model.

\section{Theoretical Description of the Basis of Wavelength Modulation Spectroscopy}

Although they are basically identical, the trace-gas detection methods that use current-modulated semiconductor lasers are generally separated into two categories, wavelength modulation (WM) and FM techniques, depending on the order of magnitude of the modulation frequency in comparison with the width of the considered absorption feature. Theoretical descriptions of these methods are also based on strongly different formalisms.

FM techniques are characterized by a modulation frequency $f$ that is much higher than the half-width of the absorption feature $\Delta v_{\text {line }}\left(f \gg \Delta v_{\text {line }}\right)$ and a small FM amplitude $\Delta v\left(\Delta v \ll \Delta v_{\text {line }}\right)$. These techniques, pioneered by Bjorklund, ${ }^{25}$ have been described theoretically in detail by several authors. ${ }^{25-28}$ These theoretical models describe the first- (and sometimes

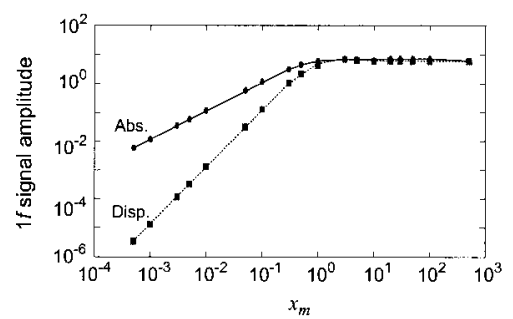

Fig. 1. Amplitude of the $1 f$ absorption and dispersion signals as a function of the normalized modulation frequency $x_{m}=f / \Delta v_{\text {line. }}$ A pure FM is considered with a FM index $\beta=\Delta v / f=1$.

also the second-) harmonic signal by considering the interaction of the modulated electric field of the laser with an absorption line. The absorption line is described by a transfer function taking into account both the absorption of the sample and its associated dispersion (a dispersion is always associated with an absorption through the Kramers-Kronig relations). Owing to the low number of sidebands present in the electric field spectrum, the signals at the first two harmonics can be determined by summation of the contributions of all sidebands after their interaction with the absorbant-dispersive sample. In this formalism the effect of the residual amplitude modulation of the laser is easily taken into account with an arbitrary IM-FM phase shift. Whereas both the absorption and the dispersion of the sample generally contribute to the detected signals, it has been shown that, for some particular values of the detection phase, a signal due only to the absorption (or the dispersion) can be obtained. ${ }^{29}$

On the other hand, WM techniques are characterized by a modulation frequency that is much smaller than the half-width of the absorption profile $(f \ll$ $\left.\Delta v_{\text {line }}\right)$. Near atmospheric pressure, the absorption lines usually extend over several gigahertz, ${ }^{30}$ making WM techniques easier to implement than FM techniques; WM techniques are particularly well adapted to these conditions. Although WM techniques can be described in principle by the same formalism as FM techniques, the calculation of the various harmonic signals is extremely tedious owing to the high number of sidebands in the laser spectrum over which the summation has to be performed. For the modulation frequencies generally used in WMS (some kilohertz or some tens of kilohertz) and for gases at atmospheric pressure, this number is of the order of $10^{5}-10^{6}{ }^{21}$ Thus WMS descriptions are preferably based on an intensity representation of the optical wave, so that no phase information is taken into account. Only the absorption of the sample is considered, and the effect of the associated dispersion is neglected. This commonly considered assumption is justified when one looks at Fig. 1, which shows the amplitudes of the absorption signal $s_{1, \text { abs }}$ at the first harmonic ( $1 f$ absorption signal) and the $1 f$ dispersion signal $s_{1 \text {,disp }}$ as a function of the normalized modulation frequency $x_{m}=f / \Delta v_{\text {line }}$. Whereas these two signals are of comparable amplitude when $x_{m} \gg 1$, 
the dispersion signal decreases much more rapidly than the absorption component for $x_{m} \ll 1$. In that case, their ratio is given approximately by the following expression:

$$
\frac{s_{1, \mathrm{abs}}}{s_{1, \mathrm{disp}}} \cong 0.9 x_{m}{ }^{-1} .
$$

The modulation frequencies used in WMS are generally between some kilohertz and some tens of kilohertz, whereas the FM amplitude $\Delta v$ is of the same order of magnitude as the width of the analyzed absorption feature, i.e., several gigahertz at atmospheric pressure. In this case, the normalized modulation frequency is extremely small $\left(x_{m} \simeq\right.$ $10^{-5}-10^{-6}$ ), and, according to expression (1), the dispersion signal becomes totally negligible in comparison with those produced by the absorption of the sample. Consequently, only the absorption of the sample must be taken into account, and a model in amplitude is not necessary. A description in intensity is sufficient in this case and is commonly used to determine the WMS signals. This description is based on the instantaneous laser frequency

$$
v(t)=v_{0}-\Delta v \cos (\omega t+\Psi),
$$

where $v_{0}$ is the optical carrier frequency, $\Delta v$ is its maximum deviation, $\omega=2 \pi f$ is the modulation angular frequency, and $\Psi$ is the phase shift between the IM and the FM. In previous descriptions of WM or FM techniques, this phase shift was often introduced in the IM rather than in the FM of the laser. ${ }^{21,22,26-28}$ We prefer to introduce it in the FM, as it describes more precisely the experimental conditions. Indeed, when the laser injection current $i$ is modulated at an angular frequency $\omega$,

$$
i(t)=i_{0}+\Delta i \cos \omega t,
$$

the light intensity $I$ is simultaneously modulated:

$$
I(t)=I_{0}+\Delta I \cos \omega t,
$$

whereas the modulation of the light frequency is phase shifted according to expression (2). A negative sign is chosen in the expression of the instantaneous laser frequency [Eq. (2)] to obtain a zero phase shift at low modulation frequencies.

In WMS the instantaneous laser frequency interacts with an absorption feature and is converted into an IM, which is measured with a photodetector. In addition, the laser frequency is slowly scanned through the absorption line at an angular frequency $\Omega$ of some hertz, and the signal at a given harmonic $n f(n=1,2, \ldots)$ of the modulation frequency is measured with lock-in detection.

The theoretical model we have developed is based on Arndt's description ${ }^{17}$ for the case of pure FM, briefly outlined in Appendix A. The effects of IM was added to this model to extend it to the more general case of a combined IM-FM. This allowed us to explain the distortion of the signal in comparison with the case of pure FM and particularly the effect of the IM-FM phase shift.

\section{Analytical Representation for a Combined Intensity Modulation-Frequency Modulation}

In the general case of a combined IM-FM, the IM is taken into account by introduction of a linear variation of the incident optical power as a function of the frequency:

$$
I_{0}(x)=I_{0}\left(p \Delta v_{\text {line }} x+1\right) .
$$

In this expression, $I_{0}$ is the optical power at the line center, and the slope $p$ describes the laser power variation as a function of the optical frequency (in inverse centimeters). This coefficient is negative in the case of a DFB laser as the optical power decreases with the laser frequency but can be positive in other types of laser. The light transmitted through a weakly absorbing sample is given by

$$
\begin{aligned}
t(x) & =I_{0}(x) \exp [-a(x)] \cong I_{0}(x)[1-a(x)] \\
& =I_{0}\left(p \Delta v_{\text {line }} x+1\right)[1-a(x)] .
\end{aligned}
$$

The efficiency of the FM depends on the modulation frequency, ${ }^{15}$ so that the $p$ coefficient takes a different value when either the sine modulation at the angular frequency $\omega$ or the low-frequency ramp used to scan the laser line through the absorption feature is considered. Two different coefficients $p_{\omega}$ and $p_{\Omega}$ are therefore introduced to describe the laser optical power variation. The first one is related to the modulation frequency $\omega$, and the second is related to the low-frequency ramp at $\Omega$. For the Lorentzian lineshape function given by Eq. (A8) in Appendix A, the detected signal is described by

$$
\begin{aligned}
s_{\mathrm{IM}-\mathrm{FM}}(x)= & I_{0}\left(p_{\Omega} \Delta \nu_{\text {line }} x_{0}-p_{\omega} \Delta \nu_{\text {line }} m \cos \omega t+1\right) \\
& \times\left\{1-\frac{a_{0}}{1+\left[x_{0}-m \cos (\omega t+\Psi)\right]^{2}}\right\} .
\end{aligned}
$$

Following a procedure similar to those proposed by Arndt in his model for pure FM, a new expression of the signal is obtained, in which the contribution of the different harmonics clearly appears:

$$
\begin{aligned}
s_{\mathrm{IM}-\mathrm{FM}}(x)= & I_{0}\left[\sum_{n=0}^{\infty} s_{n p}(x) \cos n \omega t\right. \\
& \left.-\sum_{n=0}^{\infty} s_{n q}(x) \sin n \omega t\right] .
\end{aligned}
$$

The main steps of this development are given in Appendix B. The amplitudes of the in-phase and 
quadrature terms at the frequency $n \omega$ are, respectively, given by

$$
\begin{aligned}
s_{n p}(x)= & I_{\Omega}(x) \cos n \Psi s_{n}(x)-p_{\Omega} \Delta \nu_{\text {line }} \frac{m}{2}\left(\frac{\epsilon_{n}}{2}-\epsilon_{n}+1\right) \\
& \times \cos n \Psi s_{n+1}(x)-p_{\omega} \Delta \nu_{\text {line }} \frac{m}{2}\left\{\left(\frac{2}{\epsilon_{n-1}}\right)\right. \\
& \times \cos [(n-1) \Psi] s_{n-1}(x)+\left(\epsilon_{n}-1\right) \cos [(n \\
& \left.+1) \Psi] s_{n+1}(x)\right\}, \\
s_{n q}(x)= & I_{\Omega}(x) \sin n \Psi s_{n}(x)-p_{\Omega} \Delta \nu_{\text {line }} \frac{m}{2}\left(\frac{\epsilon_{n}}{2}-\epsilon_{n}+1\right) \\
& \times \sin n \Psi s_{n+1}(x)-p_{\omega} \Delta \nu_{\text {line }} \frac{m}{2}\left\{\left(\frac{2}{\epsilon_{n-1}}\right)\right. \\
& \times \sin [(n-1) \Psi] s_{n-1}(x)+\left(\epsilon_{n}-1\right) \sin [(n \\
& \left.+1) \Psi] s_{n+1}(x)\right\},
\end{aligned}
$$

where

$$
I_{\Omega}(x)=p_{\Omega} \Delta v_{\text {line }} x+1 .
$$

The functions $s_{n}(x)$ within Eqs. (9) correspond to the case of pure FM and are given by the expressions (A9) and (A11). The signals at the first three harmonics are given for a combined IM-FM by

$$
\begin{gathered}
\left\{\begin{aligned}
s_{1 p}(x)= & I_{\Omega}(x) \cos \Psi s_{1}(x)-p_{\omega} \Delta v_{\text {line }} \frac{m}{2} \\
& \times\left[2 s_{0}(x)+\cos 2 \Psi s_{2}(x)\right] \\
s_{1 q}(x)= & I_{\Omega}(x) \sin \Psi s_{1}(x)-p_{\omega} \Delta v_{\text {line }} \frac{m}{2} \\
& \times\left[\sin 2 \Psi s_{2}(x)\right]
\end{aligned}\right. \\
\left\{\begin{aligned}
s_{2 p}(x)= & I_{\Omega}(x) \cos 2 \Psi s_{2}(x)-p_{\omega} \Delta v_{\text {line }} \frac{m}{2} \\
& \times\left[\cos \Psi s_{1}(x)+\cos 3 \Psi s_{3}(x)\right] \\
s_{2 q}(x)= & I_{\Omega}(x) \sin 2 \Psi s_{2}(x)-p_{\omega} \Delta v_{\text {line }} \frac{m}{2} \\
& \times\left[\sin \Psi s_{1}(x)+\sin 3 \Psi s_{3}(x)\right] \\
s_{3 p}(x)= & I_{\Omega}(x) \cos 3 \Psi s_{3}(x)-p_{\omega} \Delta v_{\text {line }} \frac{m}{2} \\
& \times\left[\cos 2 \Psi s_{2}(x)+\cos 4 \Psi s_{4}(x)\right] \\
s_{3 q}(x)= & I_{\Omega}(x) \sin 3 \Psi s_{3}(x)-p_{\omega} \Delta v_{\text {line }} \frac{m}{2} \\
& \times\left[\sin 2 \Psi s_{2}(x)+\sin 4 \Psi s_{4}(x)\right]
\end{aligned}\right.
\end{gathered}
$$

These general expressions for a combined IM-FM collapse to Arndt's pure FM results when no IM is considered. For pure FM, we have $p_{\omega}=p_{\Omega}=0$ and $I_{\Omega}=$ 1 , which leads to $s_{n p}(x)=\cos n \Psi s_{n}(x)$ and $s_{n q}(x)=\sin$ $n \Psi s_{n}(x)$.
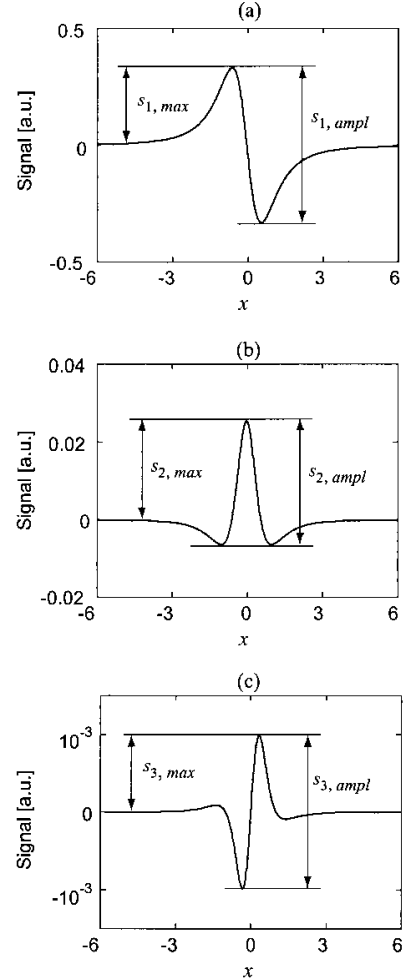

Fig. 2. Theoretical WMS signals at the first three harmonics, showing the derivativelike form of the signals. A pure FM is considered with $m=0.1$. The maximum $s_{n \text {, max }}$ and the amplitude $s_{n, \text { ampl }}$ of the signals are defined for each harmonic.

In the case of small modulation amplitudes $(m \ll$ 1 ), the signal at the $n$th harmonic is no longer simply proportional to the $n$th derivative of the lineshape function $s_{n}$, as in the case of pure FM, but derivatives of lower $\left(s_{n-1}\right)$ and upper $\left(s_{n+1}\right)$ orders are present, too. However, when $p_{\omega}=p_{\Omega}=p$ and $\Psi=0$, the signals correspond to the successive derivatives of the function $t(x)$ given in expression (6), which represents a Lorentzian line-shape function on a linear background. In all other cases, the WMS signals are no longer directly proportional to the derivatives of the function $t(x)$. Lock-in detection allows us not only to measure the in-phase and quadrature signals but also an arbitrary intermediate detection phase $\Phi_{n}$, i.e., the signals in $\cos \left(n \omega t+\Phi_{n}\right)$. The signal at the $n$th harmonic and at the detection phase $\Phi_{n}$ is given by

$$
s_{n, \Phi}(x)=I_{0}\left[s_{n p}(x) \cos \Phi_{n}+s_{n q}(x) \sin \Phi_{n}\right] .
$$

\section{Analysis of the Theoretical Model}

The WMS signals obtained in the general case of a combined IM-FM and a Lorentzian line-shape function have been calculated from expressions (11), (12), and (A11) of our theoretical model. The effect of several parameters on these signals is presented. An analysis of the WMS signals obtained in the case of a pure FM has already been reported in several papers. ${ }^{17-19,22}$ It is well known that the signal at the $n$th harmonic of the modulation frequency coincides 

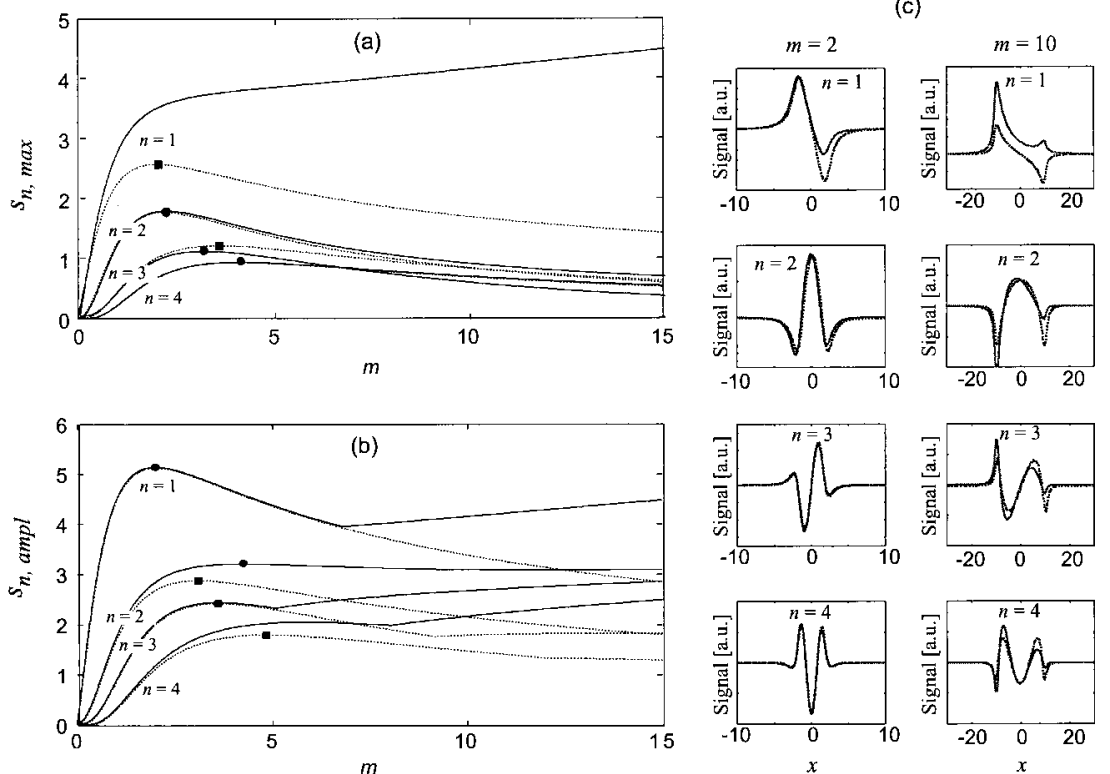

Fig. 3. Evolution of (a) the maximum and (b) the amplitude of the WMS signals at the harmonics $n=1$ to $n=4$ as a function of the modulation index $m$. Solid curves represent the case of IM-FM with $p_{\Omega}=-1\left(1 / \mathrm{cm}^{-1}\right), p_{\omega}=-2\left(1 / \mathrm{cm}^{-1}\right)$, and $\Psi=-90^{\circ}$, whereas the dashed curves represent the case of pure FM. Optimal values of $m$ are indicated by squares in the case of pure FM and by circles for IM-FM. (c) Corresponding WMS signals at the first four harmonics for $m=2$ and $m=10$. Solid curves correspond to the case of IM-FM, and dashed curves to pure FM.

with the $n$th derivative of the absorption line when $m$ $\ll 1$, whereas a distortion appears when this condition is no longer fulfilled. The maximum $\left(s_{n, \max }\right)$ and the amplitude $\left(s_{n, \text { ampl }}\right)$ of the signal at frequency $n f$, which are defined in Fig. 2, depend on the modulation index $m$. Two optimal values for $m$ at each harmonic can be obtained, for which either $s_{n \text { max }}(m)$ or $s_{n, \text { ampl }}(m)$ is maximal. These values are denoted as $m_{n, \max }{ }^{\text {opt }}$ and $m_{n \text {,ampl }}{ }^{\text {opt }}$. In the case of a pure FM, the values of $m_{n \text {, } \max }{ }^{\text {opt }}$ are $2.00,2.20,3.59$, and 4.12 for the first four harmonics, in agreement with values reported in the literature.22 For a pure FM, the values of $m_{n \text {,ampl }}{ }^{\text {opt }}$ are identical to those of $m_{n, \max }{ }^{\text {opt }}$ for odd $n$, as the signals are exactly antisymmetric in this case. However, for even values of $n$, the values of $m_{n, \text { ampl }}{ }^{\text {opt }}$ are different from $m_{n, \max }{ }^{\text {opt }}$ and are, respectively, 3.09 and 4.85 for $n=2$ and 4 .

\section{A. Effect of the Intensity Modulation}

In the case of a combined IM-FM, $p_{\Omega} \neq p_{\omega}$ and $\Psi \neq$ 0 . This produces a distortion of the signals, which no longer represent exactly the derivatives of the absorption line, even in the case of small modulation indices. This distortion is manifested in particular through an asymmetry at all the harmonics and through an offset in the first harmonic signal. The signals' asymmetry increases with $p_{\omega}$ and also depends on the IM-FM phase shift $\Psi$ and on the detection phase $\Phi_{n}$. The optimal values of the modulation index for which the signals reach their maximum $\left(m_{n, \max }{ }^{\text {opt}}\right)$ also depend on the IM parameters. Depending on these parameters, the situation can be strongly different from the well-known case of pure FM previously described. Figure 3 il- lustrates an example in the case in which $p_{\Omega}=-1$ $\left(1 / \mathrm{cm}^{-1}\right), p_{\omega}=-2\left(1 / \mathrm{cm}^{-1}\right)$, and $\Psi=-90^{\circ}$. In this example the curves of $s_{n \text {, max }}(m)$ and $s_{n \text {,ampl }}(m)$ are similar to the case of pure FM for only small values of $m$. For higher values, a strong difference between both cases is observed. Whereas the curves at all the harmonics present the same general behavior in the case of pure FM (they reach a maximum and then decrease monotonously), the situation is different in the IM-FM case considered here. For example, the curve of $s_{1, \max }(m)$ increases monotonously and does not present any maximum (at least until $m=15$ ). Furthermore, the curves $s_{1, \max }(m), s_{3, \text { ampl }}(m)$, and $s_{4, \text { ampl }}(m)$ present a first maximum, then decrease, and finally rise again up to values that can be larger than the first maximum [this is the case for $s_{3, \text { ampl }}(m)$ and $s_{4, \text { ampl }}(m)$, for example]. This behavior results from the fact that for high values of $m$, the WMS signals become strongly distorted in comparison with the case of pure FM, as can be seen in Fig. 3(c), which compares the WMS signals obtained for the combined IM-FM with those of pure FM. If we restrict the first part of the curves $s_{n \text {,max }}(m)$ and $s_{n \text {,ampl }}(m)$ (i.e., before they increase again), the optimum values of $m$ for each harmonic can be compared between the cases of pure FM and IM-FM with different characteristic values of the IM parameters $\left(p_{\Omega}, p_{\omega}\right.$, and $\left.\Psi\right)$. This comparison is shown in Fig. 4. For the even harmonics, indexes $m_{n \text {, max }}$ opt that maximize the signals are almost not modified from the case of pure FM, at least for realistic values of the modulation parameters. In contrast, they strongly vary in the odd harmonics owing to the asymmetry produced by IM. In this case, the index $m_{n \text {,ampl }}$ opt that maximizes the 

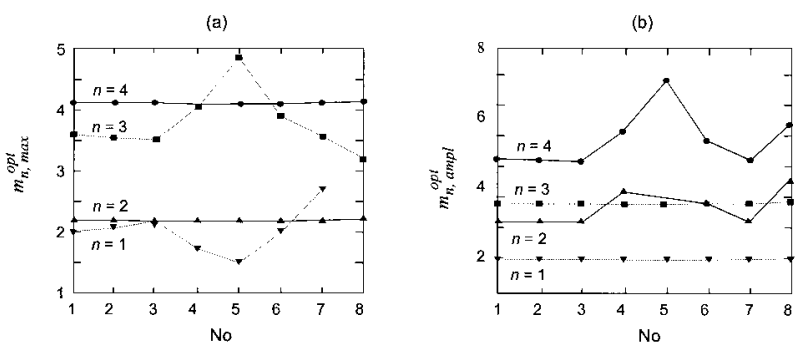

(c) \begin{tabular}{|c|c|c|c|c|c|c|c|c|}
\hline Parameter No & 1 & 2 & 3 & 4 & 5 & 6 & 7 & 8 \\
\hline$p_{\Omega}\left[1 / \mathrm{cm}^{-1}\right]$ & 0 & -1 & -2 & -1 & -1 & -1 & -1 & -1 \\
$p_{00}\left[1 / \mathrm{cm}^{-1}\right]$ & 0 & -1 & -2 & -2 & -3 & -2 & -2 & -2 \\
$\Psi[\mathrm{deg}]$ & 0 & 0 & 0 & 0 & 0 & -30 & -60 & -90 \\
\hline
\end{tabular}

Fig. 4. Optimal values of the modulation index $m$ for which the WMS signals reach (a) their maximum and (b) their maximal amplitude at the different harmonics of the modulation frequency. These values are obtained for the IM parameters shown in (c). For some sets of parameters, curves $s_{n, \max }(m)$ or $s_{n, \text { ampl }}(m)$ may present more than one maximum. In such cases, only the first value is considered. For the parameter set 8 , the curve $s_{1, \max }(m)$ does not present any maximum in the range $0<m<15$ [see also Fig. 3(a)], so there is no point associated with this condition for $n=$ 1 in (a).

total amplitude of the signals is, however, constant when the IM increases.

\section{B. Effect of the Detection Phase}

The detection phase $\Phi_{n}$ acts on both the amplitude of the signals and their symmetry. The signals at all the harmonics are generally asymmetric in the presence of a nonzero phase shift $\Psi$. However, there is for every harmonic a value of the detection phase for which the signal is totally symmetric (for even $n$ ) or antisymmetric (for odd $n$ ) with regard to the line center.

The signal magnitude varies periodically with the detection phase $\Phi_{n}$, as shown in Fig. 5 . At the $n$th harmonic, the maximal and minimal amplitudes are reached, respectively, for the detection phases

$$
\begin{aligned}
& \Phi_{n, \text { max }}=n \Psi+k \pi, \\
& \Phi_{n, \text { min }}=n \Psi+(2 k+1) \pi / 2 .
\end{aligned}
$$

For these values, the WMS signals are determined by the introduction of Eqs. (13a) and (13b) into Eqs. (12),
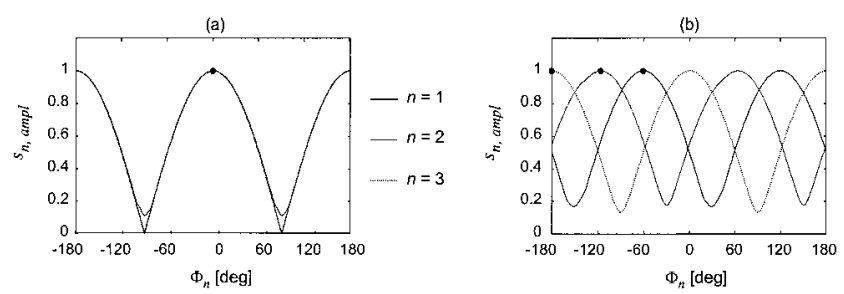

Fig. 5. Normalized amplitude $s_{n \text {,ampl }}$ of the signals at the first three harmonics as a function of the detection phase $\Phi_{n}$. The considered parameters are $m=1, p_{\Omega}=-1\left(1 / \mathrm{cm}^{-1}\right), p_{\omega}=-2$ $\left(1 / \mathrm{cm}^{-1}\right.$ ), and (a) $\Psi=0$ or (b) $\Psi=-60$ (degrees). The phase $\Phi_{n, \max }$ that maximizes the signal amplitude is indicated by a circle for each harmonic.

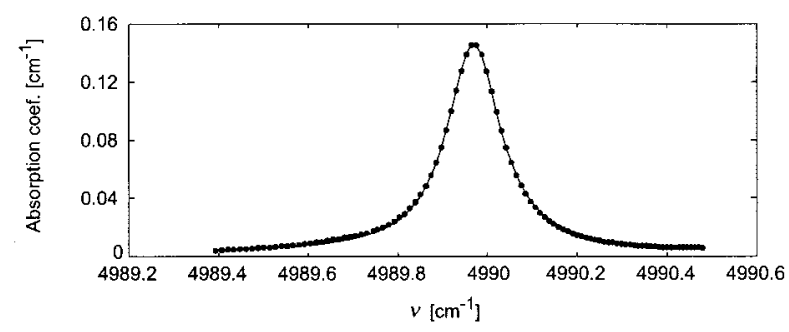

Fig. 6. Absorption line $\mathrm{R} 16$ of $\mathrm{CO}_{2}$ measured with a DFB laser emitting at $2004 \mathrm{~nm}$. The laser frequency was measured with a wavemeter with a resolution of $1 \mathrm{pm}$. Experimental points are represented by circles, and the curve results from a fit of a Lorentzian distribution.

(9a), and (9b). The corresponding signals are given by

$$
\begin{aligned}
s_{n, \Phi_{\max }}(x)= & I_{\Omega}(x) s_{n}(x)-p_{\Omega} \Delta \nu_{\text {line }} \frac{m}{2}\left(\frac{\epsilon_{n}}{2}-\epsilon_{n}+1\right) \\
& \times s_{n+1}(x) \\
& -p_{\omega} \Delta v_{\text {line }} \frac{m}{2} \cos \Psi\left[\left(\frac{2}{\epsilon_{n-1}}\right) s_{n-1}(x)\right. \\
& \left.+\left(\epsilon_{n}-1\right) s_{n+1}(x)\right], \\
s_{n, \Phi_{\min }}(x)= & p_{\omega} \Delta \nu_{\text {line }} \frac{m}{2} \sin \Psi\left[\left(\frac{2}{\epsilon_{n-1}}\right) s_{n-1}(x)\right. \\
& \left.+\left(\epsilon_{n}-1\right) s_{n+1}(x)\right] .
\end{aligned}
$$

The maximal signal at the $n$th harmonic brings in the $n$th derivative of the absorption line $\left(s_{n}\right)$ and also the lower-order $\left(s_{n-1}\right)$ and upper-order $\left(s_{n+1}\right)$ derivatives. For small modulation indices, it is dominated by the first and third terms of Eq. (14a), and the signal is mostly like an $n$th derivative of the absorption line, with an asymmetry due to the $(n-1)$ th derivative resulting from the modulation intensity. In contrast, the minimal signal at the $n$th harmonic does not bring in the $n$th derivative of the absorption line $\left(s_{n}\right)$ but only the lower-order $\left(s_{n-1}\right)$ and upper-order $\left(s_{n+1}\right)$ derivatives. For small modulation indices, it does not look like an $n$th derivative but rather as an $(n-1)$ th derivative. Furthermore, this signal is perfectly symmetric with regard to the line center for odd $n$ and antisymmetric for even $n$.

\section{Comparison with Experimental Results}

Experimental WMS signals have been measured with a DFB laser with emission frequency corresponding to an absorption line of $\mathrm{CO}_{2}$ at $\lambda=2004 \mathrm{~nm}$ (R16 line). The measurements have been performed at atmospheric pressure, at which the absorption line is well approximated by a Lorentzian line-shape function, as shown in Fig. 6. Table 1 gives the line parameters obtained experimentally and according to the HITRAN database. ${ }^{30}$ The experimental setup 
Table 1. Comparison of the Line Parameters Obtained Experimentally and According to the HITRAN Database

\begin{tabular}{lcccc}
\hline Line Parameter & Symbol & Experiment Value & HITRAN Value & Unit \\
\hline Central frequency & $\nu_{\text {line }}$ & 4989.971 & 4989.973 & $\left(\mathrm{~cm}^{-1}\right)$ \\
Linewidth & $\Delta \nu_{\text {line }}$ & 0.07324 & 0.07327 & $\left(\mathrm{~cm}^{-1}\right)$ \\
Line intensity & $S$ & $1.341 \times 10^{-21}$ & $1.328 \times 10^{-21}$ & $\left(\mathrm{~cm}^{-1} /\left(\mathrm{mol} \mathrm{cm}^{-2}\right)\right.$ \\
\hline
\end{tabular}

used is shown in Fig. 7. The laser is temperature stabilized and driven by a commercial laser driver. It is sinusoidally modulated at a frequency $f=$ $\omega / 2 \pi=11$ (kilohertz), and a current ramp is applied at $F=\Omega / 2 \pi=9.5$ (hertz) in order to scan over the absorption line. The laser beam is collimated by use of an off-axis parabolic mirror and is divided into two paths with a beam splitter. In the first arm the light beam goes through a measurement cell of length $L=$ $100 \mathrm{~cm}$ and is focused on an InGaAs photodetector. The second part of the beam is focused on a reference detector. Signals from both detectors are amplified and subtracted, in such a way that they exactly cancel out when no absorbing substance is present in the cell. The length of the two paths outside the cell is equalized, so that the absorption due to atmospheric $\mathrm{CO}_{2}$ is suppressed. This compensation technique also decreases residual nonlinearities in the intensity response and frequency response of the laser and increases the signal-to-noise ratio of the WMS signals.

The WMS signals obtained experimentally on the $\mathrm{CO}_{2}$ absorption line are compared with the theoretical signals given by the developed model [expressions (11), (12), and (A11)]. The laser modulation parameters have been determined experimentally by an interferometric method that is described elsewhere. ${ }^{29}$ Their values are indicated in Table 2 . The effect of different parameters is evaluated and compared with the theoretical predictions.

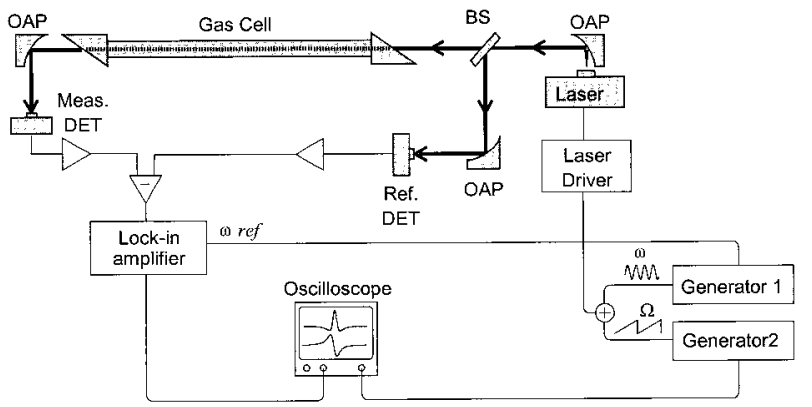

Fig. 7. Experimental setup implementing the WMS technique. $\mathrm{OAP}$, off-axis parabolic mirror; BS, beam splitter; DET, detector.

Table 2. Modulation Parameters of the DFB Laser at $f=11 \mathrm{kHz}$ and $F=9.5 \mathrm{~Hz}$

\begin{tabular}{lccc}
\hline \multicolumn{1}{c}{ Laser Parameter } & Symbol & Value & Unit \\
\hline Modulation at $F$ & $p_{\Omega}$ & -1.05 & $\left(1 / \mathrm{cm}^{-1}\right)$ \\
Modulation at $f$ & $p_{\omega}$ & -2.007 & $\left(1 / \mathrm{cm}^{-1}\right)$ \\
IM-FM phase shift at $f$ & $\Psi$ & -23 & $(\mathrm{deg})$ \\
\hline
\end{tabular}

\section{A. Effect of the Frequency Modulation}

The WMS signal measured at the second harmonic is shown in Fig. 8 as a function of the modulation index $m$. The theoretical signals given by the model for the same modulation parameters are also represented. The agreement between theoretical and experimental results is excellent, as shown by the residuals reported in each case. However, these residuals increase for higher values of $m(m>$ 2 ), so that the agreement between experimental and theoretical values becomes slightly worse. This is probably due to the nonlinear behavior of the laser optical frequency as a function of the injection current that is observed at large modulation depths. Besides, the maximal signal at the second harmonic is obtained for $m=2.20$, exactly as predicted by the model.

\section{B. Effect of the Detection Phase}

The variation of the WMS signal at the second harmonic as a function of the detection phase $\Phi_{2}$ is illustrated in Fig. 9. The theoretical signals given by the model for the same modulation parameters are also shown. An excellent agreement is again obtained between theoretical and experimental results, as shown by the residuals. The minimal amplitude of the $2 f$ signal is obtained in this case for $\Phi_{2}=44$ (degrees), which corresponds to $\Phi_{2, \min }=2 \Psi+90$
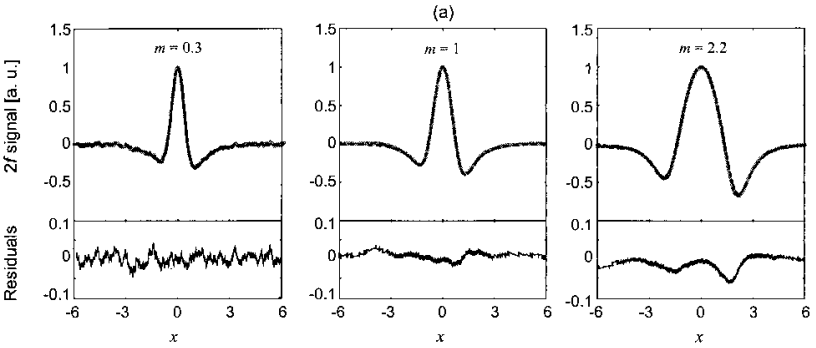

(b)
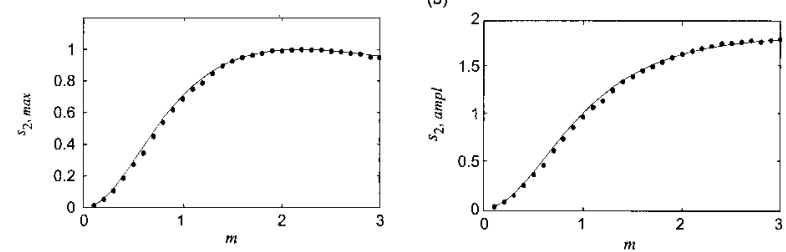

Fig. 8. (a) Normalized $2 f$ signal for different values of the modulation index $m$. Both expermental measurements and theoretical curves calculated from the model are represented, but they are indistinguishable owing to their excellent agreement. (b) Maximum $\left(s_{2, \max }\right)$ and amplitude $\left(s_{2, \text { ampl }}\right)$ of the $2 f$ signal as a function of the modulation index $m$. Circles are experimental measurements, and curves are the results of the theoretical model. 

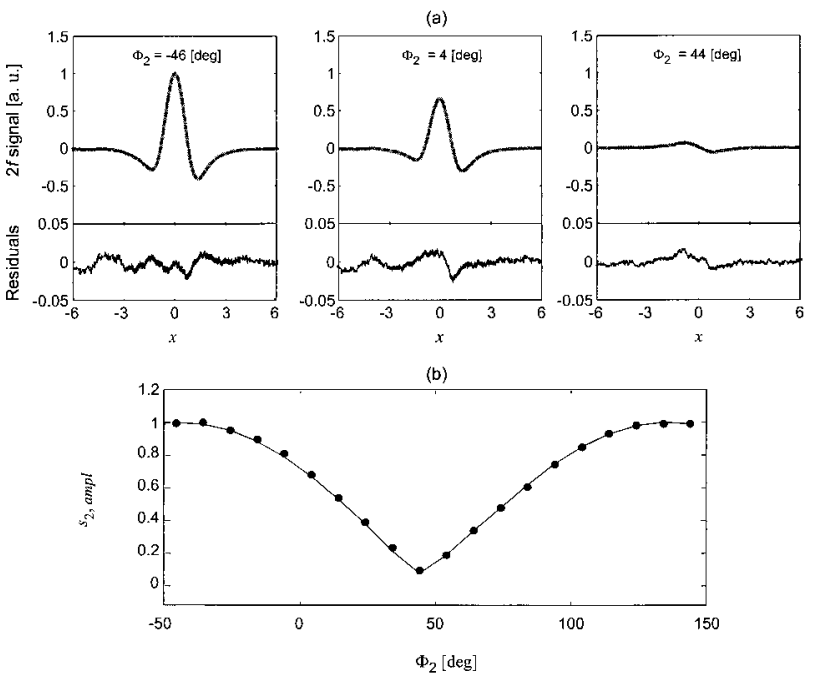

Fig. 9. (a) $2 f$ signal for different values of the detection phase $\Phi_{2}$. Both experimental measurements and theoretical curves are represented, but they are indistinguishable owing to their excellent agreement. (b) Amplitude $\left(s_{2, \mathrm{ampl}}\right)$ of the $2 f$ signal as a function of the detection phase. Circles are experimental measurements, and the curve is the result of the theoretical model.

(degrees) as predicted by expression (13b). For this value of the detection phase, the $2 f$ signal looks like a first derivative of the absorption line, in agreement with Eq. (14b).

\section{Effect of the Intensity Modulation}

The IM of the laser is characterized by the parameters $p_{\Omega}, p_{\omega}$, and $\Psi$. These values are specific to a laser and are fixed for a given modulation frequency. Their effect on the WMS signals can nevertheless be analyzed experimentally when the modulation frequency is changed. In this case the parameters $p_{\omega}$ and $\Psi$ are simultaneously modified, as both of them depend on the modulation frequency. It is therefore not possible to evaluate their effect separately. The WMS signal at the second harmonic is represented in Fig. 10 for three different values of the parameters $p_{\omega}$ and $\Psi$ and for $m=1$. The theoretical signals given by the model for the same modulation parameters are
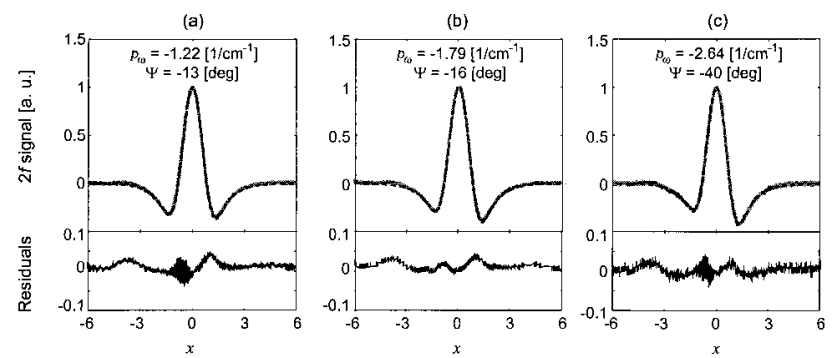

Fig. 10. Effect of the IM on the $2 f$ signal. Experimental curves (in gray) have been obtained for (a) $f=400 \mathrm{~Hz}$, (b) $f=4 \mathrm{kHz}$, and (c) $f=40 \mathrm{kHz}$. Theoretical curves calculated from the model are also represented, but they are indistinguishable from the measurements, owing to their excellent agreement. All the signals were obtained for $m=1$ and $p_{\Omega}=-1.05\left(1 / \mathrm{cm}^{-1}\right)$. also shown, as well as the residuals. For the values considered here, the effect of the parameter $p_{\omega}$ is dominant in comparison with the phase shift $\Psi$. The signal asymmetry is therefore relatively weak when $p_{\omega}$ is small, and it strongly increases with this parameter.

\section{Discussion and Conclusion}

An analytical expression of the signals generated in wavelength modulation spectroscopy has been proposed in the general case of a combined frequency modulation and intensity modulation of a laser diode. This model is based on Arndt's description of this technique and extends it to the case of a combined IM-FM with an arbitrary phase shift $\Psi$, which is the real condition when the injection current of a laser diode is modulated. The developed model considers the case of a Lorentzian absorption line. It is consequently suitable for the case of trace-gas detection at atmospheric pressure, at which the Voigt line shape is well approximated by a Lorentzian function.

The effect of several modulation parameters has been analyzed from the theoretical expressions of the WMS signals given by the model. The calculated signals have been compared with experimental WMS results measured on an absorption line of $\mathrm{CO}_{2}$ by use of a $2-\mu \mathrm{m}$ DFB laser. An excellent agreement was obtained between experimental and calculated signals, both on their shape and on their evolution as a function of the modulation parameters. These experimental results fully validate the relevance of the developed theoretical model. This description allows a better understanding of the signals generated in WMS and gives useful information for determining the optimal modulation conditions for a laser in trace-gas detection applications. Another result from the presented theoretical model is the possibility of using the WMS signals measured on a gas absorption line to determine experimentally the FM parameters of a laser (FM index and IM-FM phase shift). This issue will be addressed in the future.

\section{Appendix A: Arndt's Model for Pure Frequency Modulation}

An absorption feature described by a line-shape function $g(x)$ is considered, where

$$
x=\left(v-v_{\text {line }}\right) / \Delta v_{\text {line }}
$$

is the normalized frequency, $v_{\text {line }}$ is the center of the absorption line, and $\Delta v_{\text {line }}$ is the linewidth (halfwidth at half-maximum). For weak absorptions, the light transmitted through the sample is given by the Beer-Lambert law:

$$
t_{0}(x)=I_{0} \exp [-a(x)] \cong I_{0}[1-a(x)],
$$

where $I_{0}$ is the incident optical power and $a(x)$ represents the absorbance of the sample, which is pro- 
portional to the path length $L$, the molecules' density $N$, and the absorption line strength $S$ :

$$
a(x)=\operatorname{LNSg}(x) .
$$

In the case of a pure FM, the normalized instantaneous frequency $x$ can be written as a function of the modulation index $m=\Delta v / \Delta v_{\text {line }}$,

$$
x=x_{0}-m \cos \omega t
$$

and the instantaneous signal detected after the sample is given by

$$
s(x)=t_{0}\left(x_{0}-m \cos \omega t\right) .
$$

Following Arndt's model, ${ }^{17}$ the contribution at the different harmonics can be extracted from this signal:

$$
s(x)=\sum_{n=0}^{\infty} s_{n}(x) \cos n \omega t,
$$

with

$$
s_{n}(x)=\frac{1}{2 \pi}(-i)^{n} \epsilon_{n} \int_{-\infty}^{\infty} \mathrm{d} y J_{n}(m y) T_{0}(y) \exp (i x y),
$$

where $\epsilon_{0}=1$ and $\epsilon_{n}=2$ for $n \geq 1, J_{n}$ is the Bessel function of order $n$, and $T_{0}(y)$ is the Fourier transform of $t_{0}(x)$ :

$$
T_{0}(y)=\int_{-\infty}^{\infty} \mathrm{d} x t_{0}(x) \exp (-i x y)
$$

The integral in expression (A7) can be evaluated analytically for a Lorentzian line-shape function

$$
g(x)=\frac{1}{\pi \Delta \nu_{\text {line }}} \frac{1}{1+x^{2}},
$$

and the components of the signal at the $n$th harmonic are given in this case, according to Arndt's expression, ${ }^{17}$ by

$$
\begin{aligned}
s_{n}(x)= & I_{0}\left(J_{n}(0)-\frac{a_{0}}{2}\left\{(-i)^{n} \epsilon_{n}\right.\right. \\
& \times \frac{\left[\sqrt{(1-i x)^{2}+m^{2}}-(1-i x)\right]^{n}}{\left.\left.m^{n} \sqrt{(1-i x)^{2}+m^{2}}+\text { c.c. }\right\}\right),},
\end{aligned}
$$

where c.c. is the complex conjugate and $a_{0}$ represents the absorbance at the line center:

$$
a_{0}=a(x=0)=L N S /\left(\pi \Delta \nu_{\text {line }}\right) .
$$

The signals at the first harmonics are given by

$$
\begin{aligned}
s_{0}(x)= & I_{0}\left(1-a_{0} \frac{\sqrt{2}}{2} \frac{\sqrt{r+X}}{r}\right), \\
s_{1}(x)= & I_{0} a_{0}\left[\frac{\sqrt{2}}{m} \frac{-x \sqrt{r+X}+\operatorname{sign}(x) \sqrt{r-X}}{r}\right], \\
s_{2}(x)= & I_{0} a_{0}\left[\frac{-4}{m^{2}}+\frac{\sqrt{2}}{m^{2}}\right. \\
& \left.\times \frac{\left(r+1-x^{2}\right) \sqrt{r+X}+2|x| \sqrt{r-X}}{r}\right],
\end{aligned}
$$

$$
\begin{aligned}
s_{3}(x)= & \frac{-I_{0} a_{0}}{m^{3}}\left\{16 x+\frac{\sqrt{2}}{r}\left[x^{3}-3 x(r+1)\right] \sqrt{r+X}\right. \\
& \left.+\frac{\sqrt{2}}{r} \operatorname{sign}(x)\left(1-3 x^{2}-3 r\right) \sqrt{r-X}\right\},
\end{aligned}
$$

where

$$
X=1-x^{2}+m^{2}, \quad r=\sqrt{X^{2}+4 x^{2}} .
$$

\section{Appendix B: Developments for a Combined Intensity Modulation-Frequency Modulation}

Expression (7) can be rearranged and separated into two contributions:

$$
\begin{aligned}
s_{\mathrm{IM}-\mathrm{FM}}(x)= & g^{(1)}\left[x_{0}-m \cos (\omega t+\Psi)\right] \\
& +g^{(2)}\left[x_{0}-m \cos (\omega t+\Psi)\right],
\end{aligned}
$$

where two functions $g^{(1)}(x)$ and $g^{(2)}(x)$ are introduced:

$g^{(1)}(x)=I_{0} p_{\Omega} \Delta \nu_{\text {line }} x\left(1-a_{0} \frac{1}{1+x^{2}}\right)=p_{\Omega} \Delta \nu_{\text {line }} x t_{0}(x)$,

$g^{(2)}(x)=C I_{0}\left(1-a_{0} \frac{1}{1+x^{2}}\right)=C t_{0}(x)$,

with

$$
\begin{aligned}
C= & 1-2 p_{\Omega} \Delta \nu_{\text {line }} m \sin \Psi / 2 \sin (\omega t+\Psi / 2) \\
& +\left(p_{\Omega}-p_{\omega}\right) \Delta \nu_{\text {line }} m \cos \omega t,
\end{aligned}
$$

and $t_{0}(x)$ represents the case of pure FM according to expression (A2). The Fourier transforms of $g^{(1)}(x)$ and $g^{(2)}(x)$ are, respectively, given by

$$
\begin{aligned}
& G^{(1)}(y)=p_{\Omega} \Delta v_{\text {line }} i \frac{\mathrm{d} T_{0}(y)}{\mathrm{d} y}, \\
& G^{(2)}(y)=C T_{0}(y),
\end{aligned}
$$


where $T_{0}(y)$ is the Fourier transform of $t_{0}(x)$. Following a procedure similar to the case of pure FM, we first calculate the Fourier transform of $s_{\mathrm{IM}-\mathrm{FM}}(x)$ :

$$
\begin{aligned}
S_{\mathrm{IM}-\mathrm{FM}}(y)= & \int \mathrm{d} x s_{\mathrm{IM}-\mathrm{FM}}(x) \exp (-i x y) \\
= & \exp [-i m y \cos (\omega t+\Psi)] \\
& \times\left[G^{(1)}(y)+G^{(2)}(y)\right] .
\end{aligned}
$$

Then the time-dependent term is expanded in a Taylor series:

$$
\begin{aligned}
S_{\mathrm{IM}-\mathrm{FM}}(y)= & \frac{G^{(1)}(y) \sum_{n=0}^{\infty}(-i)^{n} \epsilon_{n} J_{n}(m y) \cos [n(\omega t+\Psi)]}{=S^{(1)}(y)} \\
& +\underbrace{G^{(2)}(y) \sum_{n=0}^{\infty}(-i)^{n} \epsilon_{n} J_{n}(m y) \cos [n(\omega t+\Psi)]}_{=S^{(2)}(y)} .
\end{aligned}
$$

The signals $s^{(1)}(x)$ and $s^{(2)}(x)$, obtained by one's taking the inverse Fourier transforms of $S^{(1)}(y)$ and $S^{(2)}(y)$, show the contributions at the different harmonics of the modulation frequency:

$$
\begin{aligned}
s^{(1)}(x)= & \frac{1}{2 \pi} \int_{-\infty}^{\infty} \mathrm{d} y S^{(1)}(y) \exp (i x y) \\
= & p_{\Omega} \Delta v_{\text {line }} i \sum_{n=0}^{\infty}(-i)^{n} \epsilon_{n} \cos [n(\omega t+\Psi)] \\
& \times \frac{1}{2 \pi} \int_{-\infty}^{\infty} \mathrm{d} y J_{n}(m y) \frac{\mathrm{d} T_{0}(y)}{\mathrm{d} y} \exp (i x y),
\end{aligned}
$$

$$
\begin{aligned}
s^{(2)}(x)= & \frac{1}{2 \pi} \int_{-\infty}^{\infty} \mathrm{d} y S^{(2)}(y) \exp (i x y) \\
= & C \sum_{n=0}^{\infty}(-i)^{n} \epsilon_{n} \cos [n(\omega t+\Psi)] \\
& \times \frac{1}{2 \pi} \int_{-\infty}^{\infty} \mathrm{d} y J_{n}(m y) T_{0}(y) \exp (i x y) .
\end{aligned}
$$

The integral within the expression of $s^{(2)}(x)$ is similar to the case of pure FM considered in Appendix A [see Eq. (A6)], whereas an integration by parts must be used for $s^{(1)}(x)$. Using trigonometric relations and grouping the different terms appearing in the summations, we then obtained expressions for $s^{(1)}(x)$ and $s^{(2)}(x)$, in which terms in phase with the current modulation (in $\cos n \omega t$ ) and in quadrature (in $\sin n \omega t$ ) are separated. Expressions (8)-(10) are finally obtained for the detected signal $s_{\mathrm{IM}-\mathrm{FM}}(x)=s^{(1)}(x)+s^{(2)}(x)$ in the case of a combined IM-FM.

\section{References}

1. D. T. Cassidy and J. Reid, "Atmospheric pressure monitoring of trace gases using tunable diode lasers," Appl. Opt. 21, 11851190 (1982).

2. D. S. Bomse, A. C. Stanton, and J. A. Silver, "Frequency modulation and wavelength modulation spectroscopies: comparison of experimental methods using a lead-salt diode laser," Appl. Opt. 31, 718-731 (1992).

3. J. Reid, B. K. Garside, J. Shewchun, M. El-Sherbiny, and E. A. Ballik, "High sensitivity point monitoring of atmospheric gases employing tunable diode lasers," Appl. Opt. 17, 1806-1810 (1978).

4. M. Loewenstein, "Diode laser harmonic spectroscopy applied to in situ measurements of atmospheric trace molecules," J. Quant. Spectrosc. Radiat. Transfer 40, 249-256 (1988).

5. K. Namjou, S. Cai, E. A. Whittaker, J. Faist, C. Gmachl, F. Capasso, D. L. Sivco, and A. Y. Cho, "Sensitive absorption spectroscopy with a room-temperature distributed-feedback quantum-cascade laser," Opt. Lett. 23, 219-221 (1998).

6. R. M. Williams, J. F. Kelly, S. W. Sharpe, J. S. Hartman, C. Gmachl, F. Capasso, D. L. Sivco, J. N. Baillargeon, and A. Y. Cho, "Spectral and modulation performance of quantum cascade lasers with application to remote sensing," in Application of Tunable Diode and Other Infrared Sources for Atmospheric Studies and Industrial Processing Monitoring II, A. Fried, ed., Proc. SPIE 3758, 11-22 (1999).

7. C. R. Webster, G. J. Flesch, D. C. Scott, J. E. Swanson, R. D. May, W. S. Woodward, C. Gmachl, F. Capasso, D. L. Sivco, J. N. Baillargeon, A. L. Hutchinson, and A. Y. Cho, "Quantumcascade laser measurements of stratospheric methane and nitrous oxide," Appl. Opt. 40, 321-326 (2001).

8. D. T. Cassidy and L. J. Bonnell, "Trace gas detection with short-external-cavity InGaAsP diode laser transmitter modules operating at $1.58 \mu \mathrm{m}$," Appl. Opt. 27, 2688-2693 (1988).

9. F. S. Pavone and M. Inguscio, "Frequency- and wavelengthmodulation spectroscopies: comparison of experimental methods using an AlGaAs diode laser," Appl. Phys. B 56, 118122 (1993).

10. M. Gabrysch, C. Corsi, F. S. Pavone, and M. Inguscio, "Simultaneous detection of $\mathrm{CO}$ and $\mathrm{CO}_{2}$ using a semiconductor diode laser at $1.578 \mu \mathrm{m}$," Appl. Phys. B 65, 75-79 (1997).

11. A. Lucchesini, I. Longo, C. Gabbanini, S. Gozzini, and L. Moi, "Diode laser spectroscopy of methane overtone transitions," Appl. Opt. 32, 5211-5216 (1993).

12. X. Zhu and D. T. Cassidy, "Modulation spectroscopy with a semiconductor diode laser by injection-current modulation," J. Opt. Soc. Am. B 14, 1945-1950 (1997).

13. A. Lucchesini, M. De Rosa, D. Pellicia, A. Ciucci, C. Gabbanini, and S. Gozzini, "Diode laser spectroscopy of overtone bands of acetylene," Appl. Phys. B 63, 277-282 (1996).

14. U. Gustafsson, G. Somesfalean, J. Alnis, and S. Svanberg, "Frequency-modulation spectroscopy with blue diode lasers," Appl. Opt. 39, 3774-3780 (2000).

15. G. Jacobsen, H. Olesen, and F. Birkedahl, "Current/frequencymodulation characteristics for directly optical frequencymodulated injection lasers at $830 \mathrm{~nm}$ and $1.3 \mu \mathrm{m}$," Electron. Lett. 18, 874-876 (1982).

16. W. Lenth, "High frequency heterodyne spectroscopy with current-modulated diode lasers," IEEE J. Quantum Electron. 20, 1045-1050 (1984).

17. R. Arndt, "Analytical line shapes for Lorentzian signals broadened by modulation," J. Appl. Phys. 36, 2522-2524 (1965).

18. J. Reid and D. Labrie, "Second-harmonic detection with tunable diode lasers-comparison of experiment and theory," Appl. Phys. B 26, 203-210 (1981).

19. J. M. Supplee, E. A. Whittaker, and W. Lenth, "Theoretical description of frequency modulation and wavelength modulation spectroscopy,” Appl. Opt. 33, 6294-6302 (1994). 
20. L. C. Philippe and R. K. Hanson, "Laser diode wavelengthmodulation spectroscopy for simultaneous measurement of temperature, pressure, and velocity in shock-heated oxygen flows," Appl. Opt. 32, 6090-6103 (1993).

21. P. Kluczynski and O. Axner, "Theoretical description based on Fourier analysis of wavelength-modulation spectrometry in terms of analytical and background signals," Appl. Opt. 38, 5803-5815 (1999).

22. P. Kluczynski, J. Gustafsson, A. Lindberg, and O. Axner, "Wavelength modulation absorption spectrometry-an extensive scrutiny of the generation of signals," Spectrochim. Acta Part B 56, 1277-1354 (2001).

23. P. Kluczynski, A. Lindberg, and O. Axner, "Background signals in wavelength-modulation spectrometry with frequency-doubled diode-laser light. I. Theory," Appl. Opt. 40, 783-793 (2001).

24. P. Kluczynski, A. Lindberg, and O. Axner, "Background signals in wavelength-modulation spectrometry by use of frequency-doubled diode-laser light. II. Experiment," Appl. Opt. 40, 794-805 (2001).

25. G. C. Bjorklund, "Frequency-modulation spectroscopy: a new method for measuring weak absorptions and dispersions," Opt. Lett. 5, 15-17 (1980).

26. W. Lenth, "Optical heterodyne spectroscopy with frequencyand amplitude-modulated semiconductor lasers," Opt. Lett. 8, 575-577 (1983).

27. M. Gehrtz, W. Lenth, A. T. Young, and H. S. Johnston, "Highfrequency-modulation spectroscopy with a lead-salt diode laser," Opt. Lett. 11, 132-134 (1986).

28. N.-Y. Chou and W. S. Sachse, "Single-tone and two-tone AM-FM spectral calculations for tunable diode laser absorption spectroscopy,” Appl. Opt. 26, 3584-3587 (1987).

29. S. Schilt, "Mesure de traces de gaz à l'aide de lasers à semiconducteur," Ph.D. dissertation, No. 2525 (Swiss Federal Institute of Technology, Lausanne, Switzerland, 2002).

30. L. S. Rothman, C. P. Rinsland, A. Goldman, S. T. Massie, D. P. Edwards, J.-M. Flaud, A. Perrin, C. Camy-Peyret, V. Dana, J. Y. Mandin, J. Schroeder, A. McCann, R. R. Gamache, R. B. Wattson, K. Yoshino, K. V. Chance, K. W. Jucks, L. R. Brown, V. Nemtchinov, and P. Varanasi, "The HITRAN molecular spectroscopic database and HAWKS (HITRAN atmospheric workstation): 1996 edition,” J. Quant. Spectrosc. Radiat. Transfer 60, 665-710 (1998). 